\title{
Interpenetrating polymer networks of polyaniline and maleimide-terminated polyurethanes
}

\author{
D.C. Liao ${ }^{\text {a }}$, K.H. Hsieh ${ }^{\text {a,* }}$, Y.C. Chern ${ }^{\text {a }}$, K.S. Ho ${ }^{\text {b }}$ \\ "Department of Chemical Engineering, National Taiwan University, Taipei 106, Taiwan \\ ${ }^{b}$ Department of Chemical Engineering. National Kaohsiung Institute of Technology, Kaohsiung 807, Taiwan
}

Received 28 October 1996; accepted 11 November 1996

\begin{abstract}
A series of novel conducting interpenetrating polymer networks (IPNs) was prepared by sequential polymerization of malcimide-terminated polyurethane (UBMI) and polyaniline (PANI) which was doped with dodecylbenzenesulfonic acid (PANIDB). From the differential scanning calorimetry (DSC) analysis of the IPNs, the compatibility of the polyether-type UBMI with PANIDB was better than that of the polyester-type UBMI with PANIDB. The IPNs exhibited higher conductivity and better mechanical properties when the polyether-type UBMIs were used in the composition. The scanning electron microscopy (SEM) of the IPNs illustrated heterogeneous structure of the cured resins when the polyester-type UBMI was incorporated in the UBMI/PANIDB IPNs. Scanning tunneling microscopy (STM) images of the compatible polyether-type PANIDB/UBMI IPNs indicated that the conducting region of the PANIDB was distributed as continuous conducting networks in the matrix, which resulted in higher conductivity and very low threshold concentration of the PANIDB for the conducting IPNs.
\end{abstract}

Keywords: Interpenetrating polymer network; Polyurcthanc; Polyaniline; Bismaleimide; Conductivity; Mechanical propertics; Morphology; Scanning tunneling microscopy

\section{Introduction}

Polyaniline (PANI) is one of the most important electrically conductive polymers, because of its inherent high conductivity $\left(\sigma=1-20 \mathrm{~S} \mathrm{~cm}^{-1}\right)$ and high environmental stability [1-3]. Moreover, the monomer aniline is relatively inexpensive. The polymerization is straightforward and proceeds with high yield. Therefore, PANI has been considered as one of the most promising conducting polymers [4-6]. However, processing of PANI is difficult. Melt processing is not possible, since PANI decomposes below its softening or melting temperature $[7,8]$. Although the undoped form of PANI is soluble in various solvents such as tetrahydrofuran, dimethylforamide or $N$-methyl-2-pyrrolidone, the conducting form (doped) of the polymer is insoluble in almost all solvents (except concentrated sulfuric acid and other strong acids) $[9,10]$; therefore, it is categorized as an intractable and unprocessible material [7]. Several studies by modifying the polymer chain with various benzene rings or $N$-substituents have been attempted to improve the processibility of PANI, but the degree of conjugation and coplanarity in the modified PANI is usually less than that of the PANI homo-

\footnotetext{
* Corresponding author. Tel.: +8862 363 5230; fax: +88623623040.
}

polymer. This would reduce the conductivity by several orders in magnitude more than the PANI homopolymer [3]. On the other hand, there have been some reports on the fabrication of conducting polyblends which are co-soluble in the same solvent; the electrical conductivities of the resultant blends were usually relatively low, and most of them had a rather high threshold concentration [11-13].

In this study, the concept of interpenetrating polymer networks (IPNs) was adopted to improve the processibility and mechanical properties of PANI-containing IPNs. In a previous article, a series of novel urethane-modified bismaleimides (UBMIs) was prepared through the imidization of the NCOterminated polyurethane prepolymer and maleic anhydride [14]. The thermal polymerization of the neat UBMI could be carried out in the temperature range of $102-245^{\circ} \mathrm{C}$, and the UBMIs with lower chain length could be crosslinked and reacted faster at lower temperatures. The crosslinked UBMIs behave as a rubber-like material. In this work, the novel conducting IPNs from PANI doped with dodecylbenzene sulfonic acid (DBSA) and UBMI were prepared by using a sequential polymerization technique. The mechanical propcrtics, glass transition temperature, conductivity and morphology of the conducting IPNs were investigated and are discussed. 


\section{Experimental}

\subsection{Materials}

Poly (tetramethylene oxide) diols (PTMOs) with molecular weight 650 (PTMO650) and 1000 (PTMO1000), and poly (butylene adipate) diol (PBA) with molecular weight 1000 (PBA1000) were used as polyols. 4,4'-Diphenylmethane diisocyanate (MDI) was used as the isocyanate for the polyurethane (PU) preparation. Benzoyl peroxide was the initiator for the polymerization of the unsaturated maleimide groups. Polyols were continuously stirred and degassed overnight at $70^{\circ} \mathrm{C}$ in the flask before they were used. MDI, maleic anhydride, aniline and ammonium persulfate were employed as received.

\subsection{Preparation of $P U$ prepolymer}

To prepare PU prepolymer, two equivalents of MDI were charged into a reaction kettle and heated until molten at about $55^{\circ} \mathrm{C}$. Then, one equivalent of polyol was added and mixed with the molten MDI. The mixture was vigorously agitated by a mechanical stirrer. The reaction took place under a dry nitrogen atmosphere at a temperature of approximately 68 ${ }^{\circ} \mathrm{C}$. When the $-\mathrm{NCO}$ content reached the theoretical value, the reaction was stopped.

\subsection{Synthesis of maleimide-terminated polyurethanes (UBMIs) [14]}

The maleimide-terminated polyurethanes (UBMI) were synthesized by the condensation reaction of maleic anhydride and NCO-terminated PU prepolymer with a 2:1 molar ratio according to the following reaction:
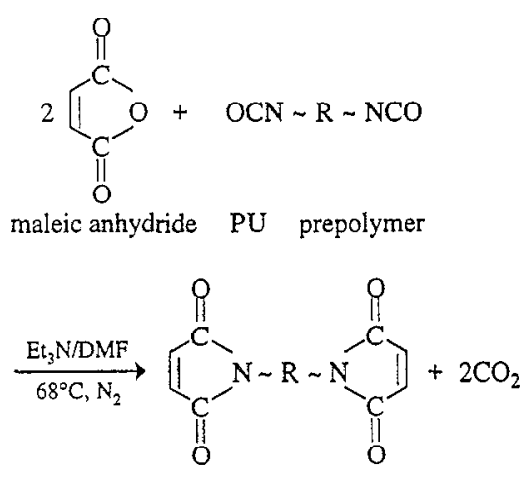

UBMI

$\mathrm{R}$ is the urethane-terminated polyether or polyester diol. The reactions were carried out in a three-necked flask which was equipped with a stirring device, reflux condenser, thermometer, heating mantle and constant flow of dry nitrogen. The NCO-terminated PU prepolymer, dissolved in chloroform, was added to the solution of maleic anhydride and triethyl amine (TEA) catalyst at $68^{\circ} \mathrm{C}$. The evolution of $\mathrm{CO}_{2}$ gas was observed at the start of the reaction. During this period, samples were periodically drawn for IR analysis. The reaction was stopped until the $\mathrm{NCO}$ absorption peak in $\mathrm{IR}$ at $2270 \mathrm{~cm}^{-1}$ and the $\mathrm{C}=\mathrm{O}$ (of acid anhydride) absorption peak at $184.8 \mathrm{~cm}^{-1}$ disappeared. The products dissolved in chloroform produced a dark-brown solution. The detailed identification and properties of the UBMIs were given in a previous paper [14].

\subsection{Preparation of PANI}

To prepare PANI powder, the aniline monomer $(80 \mathrm{ml})$ was added to $500 \mathrm{ml}$ of $1 \mathrm{M}$ hydrochloric acid $(\mathrm{HCl})$ and stirred in an ice bath. Ammonium persulfate $\left(\left(\mathrm{NH}_{4}\right)_{2} \mathrm{O}_{8}, 46\right.$ g) was then added slowly to the aniline solution and stirred for $1.5 \mathrm{~h}$ in the ice bath with temperature controlled between 0 and $5^{\circ} \mathrm{C}$, and then $2.5 \mathrm{~h}$ at room temperature. The precipitate was collected and washed with $1 \mathrm{M} \mathrm{HCl}$ solution until the filtrate was clean. Then the precipitate was washed with distilled water and collected [15].

The collected precipitate was first neutralized by 11 of $0.1 \mathrm{M}$ ammonium hydroxide $\left(\mathrm{NH}_{4} \mathrm{OH}\right)$ solution and then the precipitate was collected. Then 11 of $0.1 \mathrm{M}$ ammonium hydroxide solution was added to the precipitate and the mixture was left with stirring for $15 \mathrm{~h}$. After filtration and washing by distilled water, the precipitate was dried under vacuum and ground into powder as emeraldine base (EB).

\subsection{Doping of PANI by DBSA}

The EB powder was mixed at room temperature with DBSA in chloroform. The molar ration of DBSA to PANI was 0.7 . After stirring for $24 \mathrm{~h}$, the mixture dissolved to yield viscous, dark-green solutions. The frec-standing films could be obtained after casting the solution at room temperature. In order to remove any dopant (DBSA) that was not complexed with the polymer, the films were then rinsed in water at 70 ${ }^{\circ} \mathrm{C}$. The doped samples were dried and reweighed to determine the amount of dopant uptake. Fully DBSA-doped PANI (PANIDB) could be obtained following the procedure described in the literature $[7,9]$.

\subsection{Preparation of PANIDB/UBMIIPNs}

A solution of PANIDB and UBMI at various weight ratios in chloroform was placed in a flask. The mixture was stirred by a magnetic device for $48 \mathrm{~h}$, with the aim of approaching equilibrium conditions. Then a solution of benzoyl peroxide ( $1 \mathrm{wt} . \%$ based on the weight of UBMI) was added to the flask and stirred well at room temperature. The mixture was cast into a mold at the same temperature. After the solvent was allowed to evaporate for $10 \mathrm{~h}$, the resultant mixture, formed as a film, was cured at $70{ }^{\circ} \mathrm{C}$ for $10 \mathrm{~h}$ and then postcured at $120^{\circ} \mathrm{C}$ for $3 \mathrm{~h}$. The samples were then conditioned in a desiccator at $50 \%$ relative humidity for at least 3 days before testing. 


\subsection{Testing methods}

Differential scanning calorimetry (DSC) analysis was performed on a DuPont 9900 at a heating rate of $20^{\circ} \mathrm{C} / \mathrm{min}$ for the IPNs over a temperature range of -120 to $300^{\circ} \mathrm{C}$.

The stress-strain property was measured by a Tensilon (mode: TCF-RC, Yashima Works Ltd., Japan). The stressstrain test procedure was followed as described in ASTM D-412 with a crosshead speed of $10 \mathrm{~mm} / \mathrm{min}$ and at least five specimens were used for the test.

The conductivity of conducting films was measured using the collinear four-point probe method. The polymer film was fastened to wire contacts using conductive silver paint. A constant current was applied to the outer electrodes, and the resulting voltage across the inner electrodes was recorded (Keithley digital multimeter).

Morphological studies were performed by using scanning electron microscopy (SEM). Microphotographs were taken on the surface made by fracturing the specimen in liquid nitrogen and then casting it with Au powder. A Hitachi S800 scanning clectron microscope was used for morphological observation.

Scanning tunneling microscopy (STM) images were obtained in air by using a Nanoscope III E (Digital Instrument) with a PtIr tip. Conductive silver paint was used to

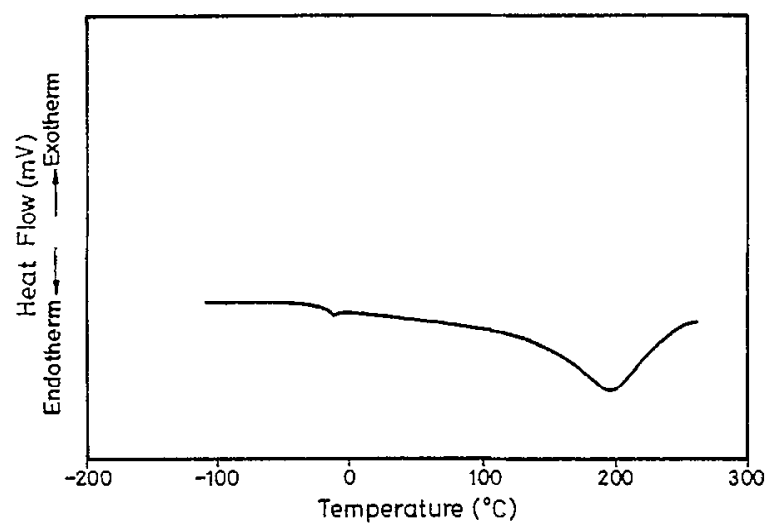

Fig. 1. DSC curve of PANIDB.

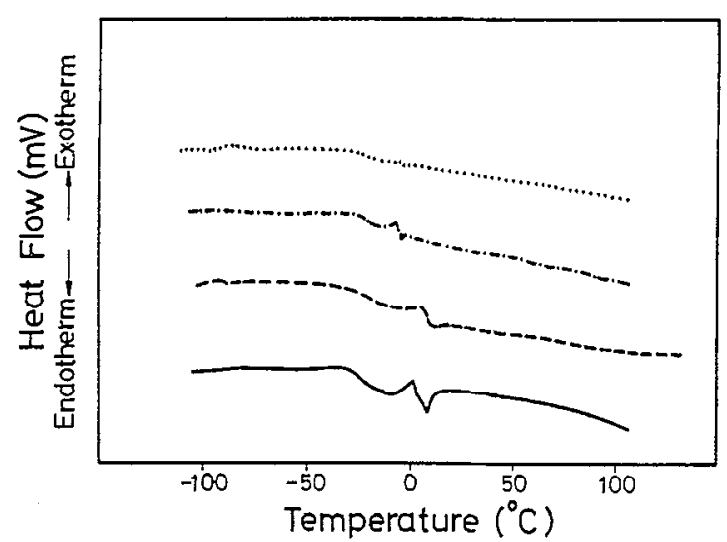

Fig. 2. DSC curves of PANIDB/UBMI(PBA1000) conducting IPNs for various PANDB/UBMI ratios: (- - $0 / 100 ;(-\longrightarrow) 10 / 90$; (-..) $50 / 50 ;(\cdots) 90 / 10$. make electrical contact with the samples. Scan speeds were generally kept at $3.8 \mathrm{~Hz}$ and the set point current was $2.0 \mathrm{nA}$. The image of the current data type was shown as computergenerated topographical rendering.

\section{Results and discussion}

\subsection{DSC analysis}

As shown in Fig. 1, no glass transition temperature was found for the neat PANIDB sample because of its rigid structure in the main chain. One endothermic peak around $200{ }^{\circ} \mathrm{C}$ can be observed; it corresponds to the degradation of the doped DBSA. The small peak at $0{ }^{\circ} \mathrm{C}$ is due to the absorbed moisture. The DSC curves of the PANIDB/UBMI(PBA1000) conducting IPNs are shown in Fig. 2. The effects of UBMI(PBA) content in the PANIDB/UBMI IPNs on the DSC curves are detected by the shift of their glass transition temperatures ( $T_{\mathrm{g}}$ values). No shift in $T_{\mathrm{g}}$ was observed for the PANIDB/UBMI(PBA) IPNs. This might indicate that a significant degree of phase separation is present in the matrix and results from inherent thermodynamic incompatibility in the component polymer networks. As shown in Figs. 3 and 4, polyether-type of PTMO polyol was employed to prepare PANIDB/UBMI(PTMO) IPNs. An increase of $T_{\mathrm{g}}$ of IPNs with increasing PANIDB content was observed. This tendency indicates that the probability of permanent entanglement and interlocking is significantly enhanced due to high compatibility when polyether-type UBMIs are employed in the IPNs. Furthermore, it was also observed that the polyether-type UBMI with PANIDB IPNs with shorter soft segments in the UBMI (i.e., PTMO650) has higher $T_{\mathrm{g}}$ than thosc with longer soft segments in the UBMI (i.e., PTMO1000). The reason for this behavior is the increased crosslink density of the UBMI due to shorter chain length of soft segments in UBMI. Moreover, when higher content of PANIDB was incorporated, the $T_{\mathrm{g}}$ of the PANIDB/UBMI(PTMO650) IPNs disappeared in the DSC curves. This means that the

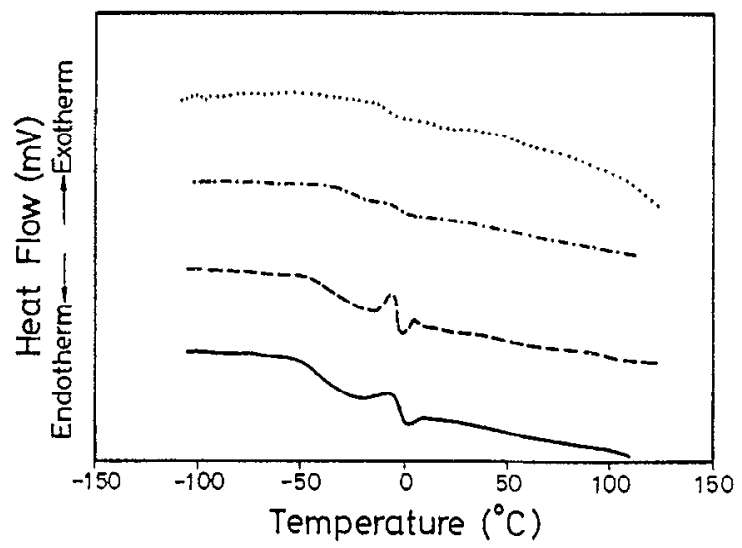

Fig. 3. DSC curves of PANIDB/UBMI(PTMO1000) conducting IPNs for various PANIDB/UBMI ratios: (- $\longrightarrow$ ) 0/100; (- $\longrightarrow$ ) 10/90; (...) $50 / 50 ;(\cdots)$ 90/10. 


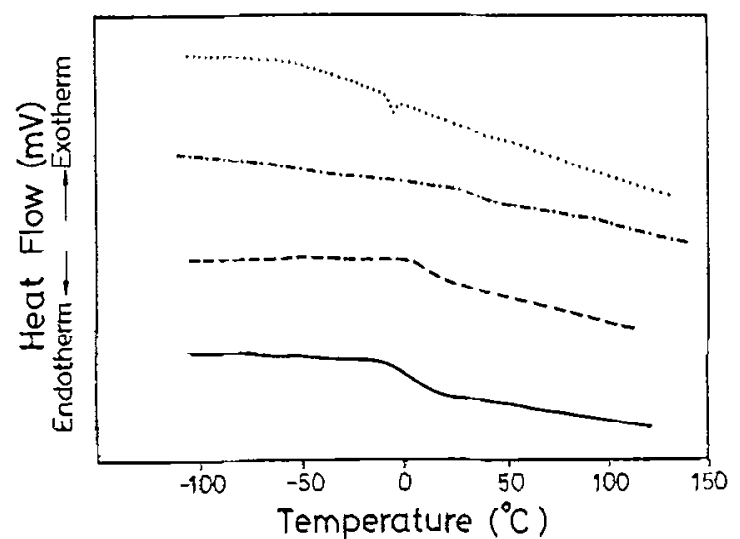

Fig. 4. DSC curves of PANIDB/UBMI(PTMO650) conducting IPNs for various PANIDB/UBMI ratios: (- $\longrightarrow$ ) $/ 100 ;(\longrightarrow-$ 10/90; $(\cdots \cdots) 50 / 50 ;(\cdots) 90 / 10$.

rigid PANIDB behaves as a continuous phase due to good compatibility with the UBMI(PTMO650), which can be considered as a homogeneous system.

\subsection{Conductivity}

The conductivity of the PANIDB/UBMI IPNs is shown in Fig. 5 and the values are listed in Table 1. The conductivity value for the neat PANIDB is $74.9 \mathrm{~S} \mathrm{~cm}^{-1}$. When the UBMI(PBA1000) was introduced, the conductivity of the IPNs significantly decreased with increasing UBMI content. When the polyether-type UBMI was employed, the conductivity of the IPNs was much higher. This result indicates that the conducting component would interpenetrate and distribute homogeneously to form a continuous network in the matrix. Therefore, the compatible PANIDB/UBMI(PTMO) IPN matrix can contribute more conductive passages through the conducting network, which results in higher conductivity.

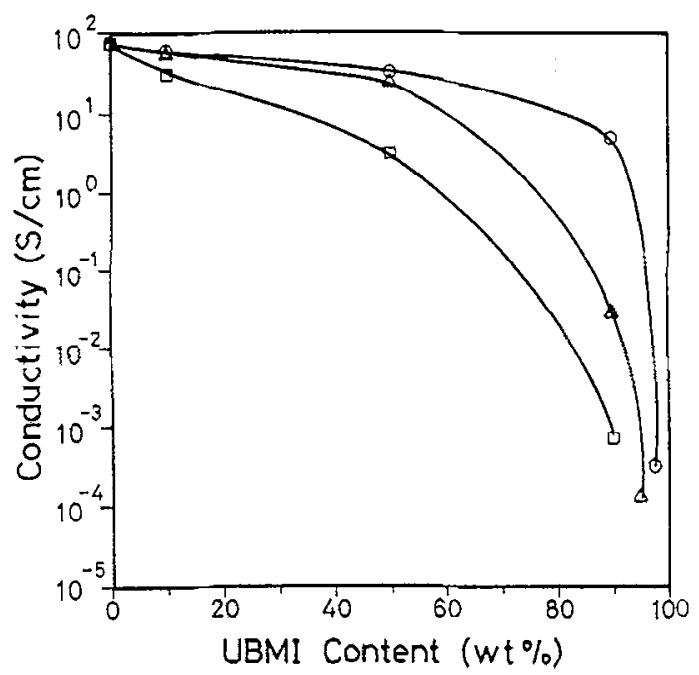

Fig. 5. Conductivity of PANIDB/UBMI conducting IPNs with various UBMI contents: (O) PANIDB/UBMI(PTMO650); ( $\triangle$ ) PANDB / UBMI(PTMO1000); ( $\square$ ) PANIDB/UBMI(PBA1000).
Furthermore, the PANIDB/UBMI(PTMO650) IPN exhibits higher conductivity $\left(4.8 \mathrm{~S} \mathrm{~cm}^{-1}\right)$ even though the content of the conducting component (PANIDB) is only $10 \mathrm{wt} . \%$. This value is higher than the conductivity of the PANIDBSA-PE polyblend [7] and is comparable to that of the PANI-CSA (camphor sulfonic acid)-PMMA polyblend, at the same PANIDB content in the system [9]. Moreover, as shown in Fig. 5, at low PANIDB content in the PANIDB/ UBMI IPNs, the conductivity of the IPNs begins to increase sharply. This behavior may be explained by dissolving a conducting material into an insulating matrix. The beginning of the transition, namely, the 'percolation threshold', is dependent on the connectivity of conducting networks and the nature of the insulating matrix. The higher the interpenetration of the two polymer networks, the higher is the conductivity. The percolation threshold concentration of the PANIDB/UBMI(PTMO) IPNs is lower than that of the PANIDB/UBMI(PBA) IPNs, i.e., in the PANIDB/UBMI IPN system, the degree of compatibility between the conducting component (PANIDB) and the insulating component (UBMI) plays an important role in the degree of mixing and dispersion of the conducting component in the matrix, which determines the threshold concentration and determines the conductivity of the PANIDB/UBMI IPNs.

\subsection{Stress-strain properties}

The stress-strain properties of both kinds of IPNs with various UBMI chain lengths and compositions are shown in Fig. 6 and the values are listed in Table 1. The tensile strength at break increases with the PANIDB content up to a maximum value, then decreases with a continuous increase of the PANIDB in the IPNs. The initial increase in tensile strength results from the interpenetrating effect, while the followed decrease is oue to more rigidity and brittleness of the PANIDB introduced. It was also observed that the PANIDB/UBMI IPNs

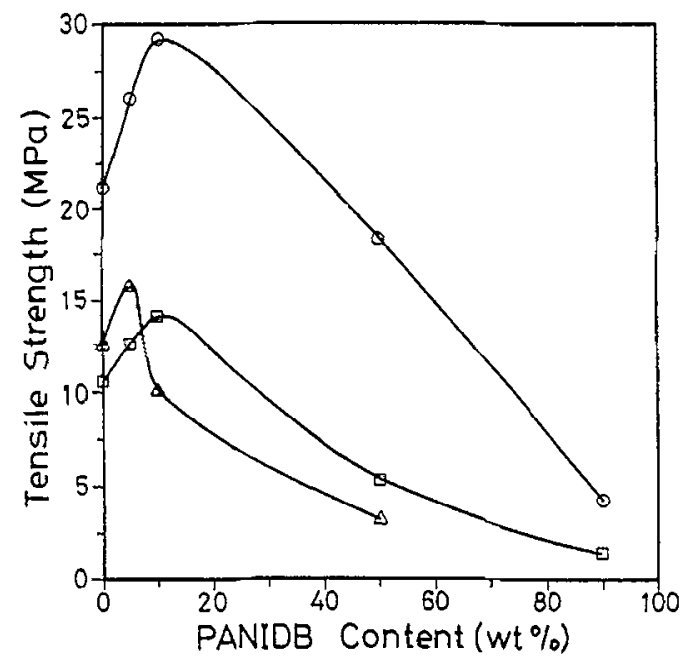

Fig. 6. Tensile strength of PANIDB/UBMI conducting IPNs with various

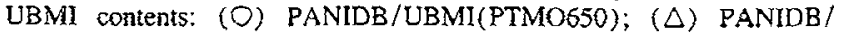
UBMI (PTMOIO00); (口) PANIDB/UBMI(PBA1000). 
Table 1

Conductivity and tensile strength of PANIDB/UBMI conducting IPNs

\begin{tabular}{lccr}
\hline IPN & UBMI content (wt.\%) & Conductivity $\left(\mathrm{S} \mathrm{cm}^{-1}\right)$ & Tensile strength (MPa) \\
\hline PANIDB/UBMI & 0 & 74.9 & \\
PANIDB/UBMI(PBA1000) & 10 & 30.6 & 3.12 \\
PANIDB/UBMI(PBA1000) & 50 & $7.0 \times 10^{-4}$ & 3.2 \\
PANIDB/UBMI(PBA1000) & 90 & & 10.1 \\
PANIDR/UBMI(PBA1000) & 100 & 59.5 & 12.6 \\
PANIDB/UBMI(PTMO1000) & 10 & 26.4 & 1.4 \\
PANIDB/UBMI(PTMO1000) & 50 & 0.029 & 5.2 \\
PANIDB/UBMI(PTMO1000) & 90 & & 14.1 \\
PANIDB/UBMI(PTMO1000) & 100 & 61.0 & 10.5 \\
PANIDB/UBMI(PTMO650) & 10 & 31.2 & 4.2 \\
PANIDB/UBMI(PTMO650) & 50 & 4.8 & 18.3 \\
PANIDB/UBMI(PTMO650) & 90 & & 29.2 \\
PANIDB/UBMI(PTMO650) & 100 & & 21.1 \\
\hline
\end{tabular}
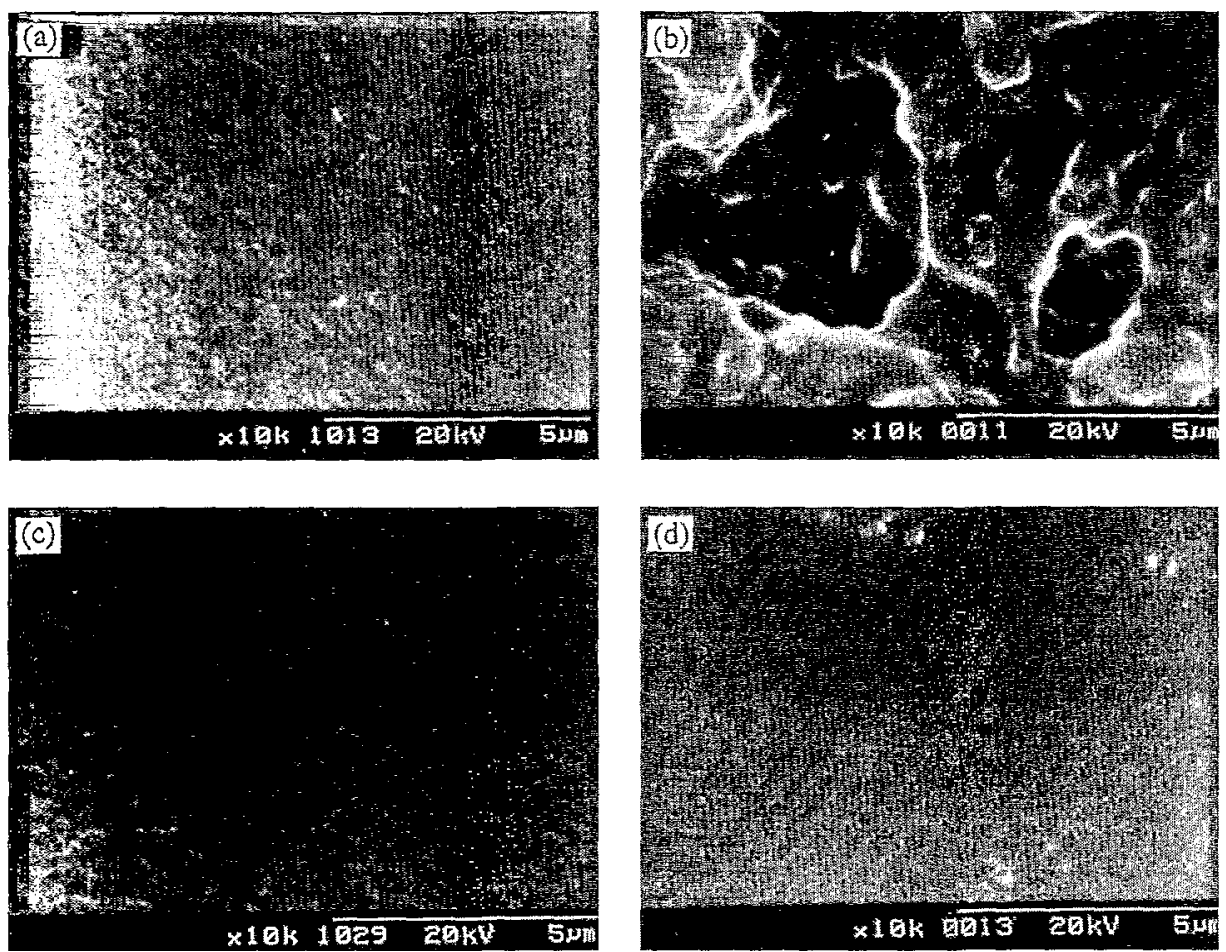

Fig. 7. SEM photomicrographs of PANIDB/UBMI conducting IPNs: (a) PANIDB/UBMI, 100/0; (b) PANIDB/UBMI(PBA1000), 50/50; (c) PANIDB/ UBMI(PTMO1000), 50/50; (d) PANIDB/UBMI (PTMO650), 50/50.

with shorter soft segments in the UBMI (i.e. PTMO650) have significantly higher tensile strength than those with longer soft segments in the UBMI. The reason for this behavior is due to the increased crosslink density in the UBMI with the shorter chains. For the PANIDB/UBMI(PBA) series, the IPNs exhibit low mechanical strength due to the lower compatibility of the resultant IPNs. However, the incorporation of UBMI in the PANIDB/UBMI IPNs could actually enhance the mechanical strength of the materials and improve processibility of the conducting materials.

\subsection{SEM}

SEM photomicrographs of the PANIDB/UBMI IPNs are shown in Fig. 7. SEM of the ncat PANIDB illustrates a homogeneous morphology. However, when polyester-type UBMI(PBA 1000) is introduced into the PANIDB/UBMI IPNs, the fractured surface in SEM shows more roughness, as shown in Fig. 7(b). This indicates a heterogeneous morphology. On the contrary, as the polyether-type UBMI(PTMO1000) or UBMI(PTMO650) is incorporated 

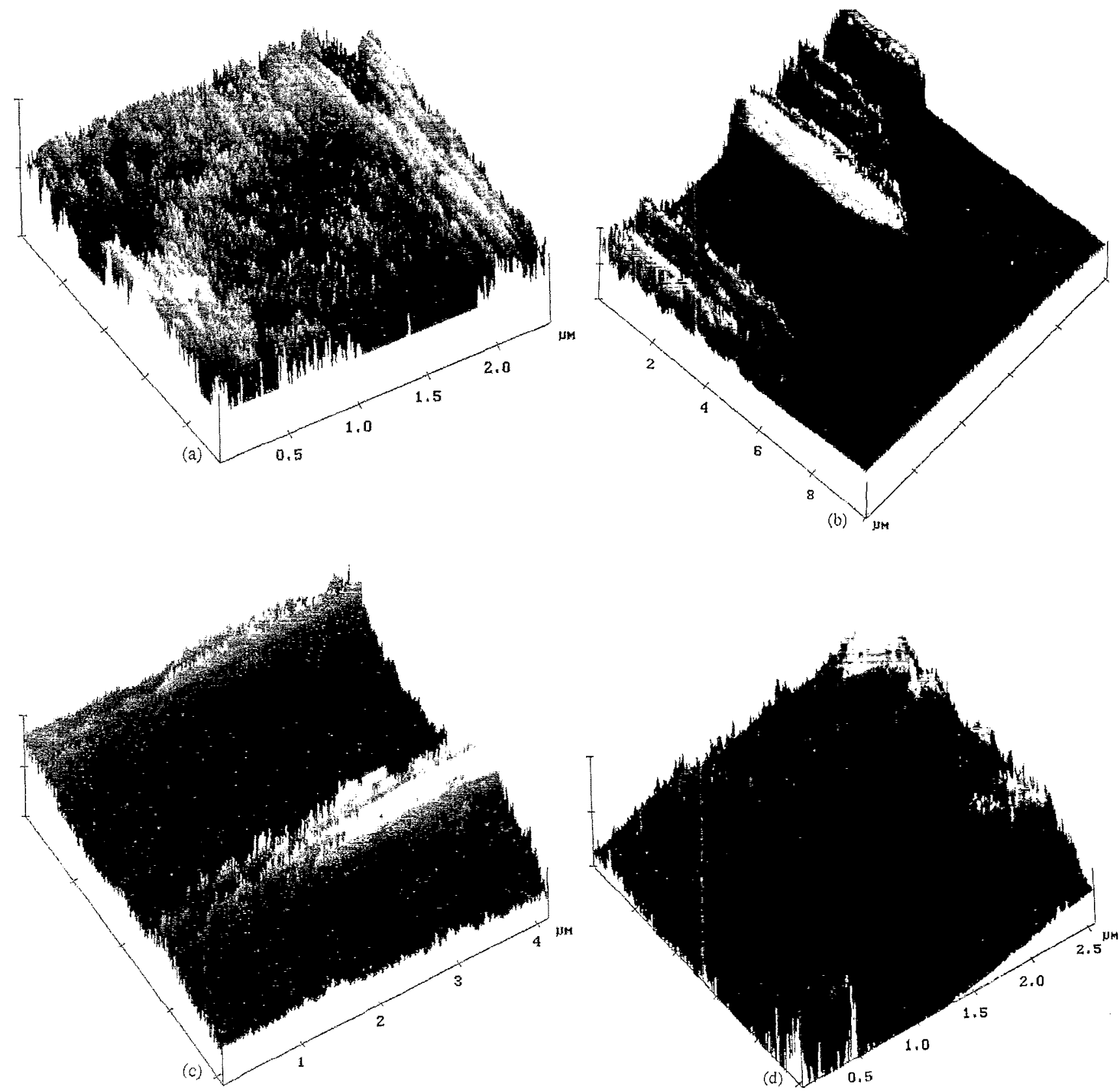

Fig. 8. STM micrographs of PANIDB/UBMI conducting IPNs: (a) PANIDB/UBMI, 100/0; (b) PANIDB/URMI(PBA1000), 10/90; (c) PANIDB/ UBMI(PTMO1000), 10/90; (d) PANIDB/UBMI(PTMO650), 10/90.

in the PANIDB/UBMI IPNs, the surface morphology is shown as a homogeneous phase in Fig. 7(c) and (d). These SEM observations are consistent with the DSC measurements, where the observed homogeneous morphology corresponds to the $T_{\mathrm{g}}$ shifting of the IPNs.

\subsection{STM}

Fig. 8 shows the STM current image of the IPNs. The STM studies offer a tentative explanation for the relationship between the observed macroscopic conductivity and the distribution of the conducting component (PANIDB) in the matrix. For the neat PANIDB (Fig. 8(a)), it is seen that a large number of high current spots are densely distributed in the matrix. They were considered as identical with the conductive regions of the PANIDB. Fig. 8(b) appears as a plateau-flat image due to the phase-separated PANIDB out of the UBMI(PBA) matrix. This indicates that the conducting PANIDB is distributed discontinuously in the IPN networks. As described previously, the polyester-type IPN series exhibits lower conductivity and higher threshold concentration; the STM image offers more evidence for these results. Moreover, Fig. 8(c) and (d) shows the STM images of the compatible PANIDB/UBMI(PTMO) IPNs of different PTMO molecular weight. The current spots exhibit a more uniform distribution for the IPNs with a lower PTMO molecular weight of 
650. This indicates that the PANIDB disperses finely and interpenetrates as continuous conducting networks in the matrix, which results in higher conductivity and lower threshold concentration for the conducting IPNs.

\section{Conclusions}

PANIDB/UBMI IPNs with polyether-type UBMI incorporated in the IPNs have better compatibility between the two polymer components. The conductivity and continuous mechanical properties of the polyether-type PANIDB/UBMI IPNs are higher than those of the polyester-type PANIDB/ UBMI IPNs. The conducting region of the PANIDB distributes uniformly and interpenetrates in the compatible IPNs, which results in high conductivity and very low threshold concentration of the PANIDB for the conducting IPNs. SEM and STM of the IPNs illustrate a heterogeneous structure of the cured resins when the polyester-type UBMI was incorporated.

\section{Acknowledgements}

The authors acknowledge with gratitude financial support from the National Science Council, Taipei, Taiwan, ROC, through Grant Nos. NSC 82-0405-E002-077 and NSC 852216-E002-004.

\section{References}

[1] J.P. Pouget, M.E. Jozefowicz, A.J. Epstein, X. Tang and A.G. MacDiarmid, Macromolecules, 24 (1991) 779.

[2] K. Mizoguchi, M. Nechtschein, J.P. Travers and C. Menardo, Phys. Rev. Lett., 63 (1989) 66.

[3] F. Lux, Polymer, 35 (1994) 2915.

[4] Y. Fong and J.B. Schlenoff, Polymer, 36 (1995) 639.

[5] K. Tzou and R.V. Gregory, Synth. Met., 47 (1992) 267.

[6] R.V. Gregory, W.C. Kimbrell and H.H. Kuhn, Synth. Met., 28 (1989) C823.

[7] C.Y. Yang, Y. Cao, P. Smith and A.J. Heeger, Synth. Met., 53 (1993) 293.

[8] Y. Cao, P. Smith and A.J. Ileeger, Synth. Met., 32 (1989) 263.

[9] Y. Cao, P. Smith and A.J. Heeger, Synth. Met., 48 (1992) 91.

[10] Y. Cao, A. Andreatta, A.J. Heeger and P. Smith, Polymer, 30 (1989) 2305.

[11] S.A. Chen and W.G. Fang, Macromolecules, 24 (1991) 1242

[12] B. Wessling and HI. Volk, Synti. Met., 18 (1987) 671.

[13] S. Ghosh and R. Chandra, J. Appl. Polym. Sci., 40 (1990) 1049.

[14] D.C. Liao and K.H. Hsieh, J. Polym. Sci., Polym. Chem., 32 (1994) 1665.

[15] A.G. MacDiarmid, J.C. Chiang, A.F. Richter and A.J. Epstein, Synth. Met., 18 (1987) 285. 